\title{
Influence of breastfeeding and postnatal nutrition on cardiovascular remodeling induced by fetal growth restriction
}

\author{
Merida Rodriguez-Lopez ${ }^{1-3}$, Lyda Osorioº ${ }^{2}$ Ruthy Acosta-Rojas', Josep Figueras' ${ }^{1}$, Monica Cruz-Lemini', Francesc Figueras ${ }^{1,4}$, \\ Bart Bijnens ${ }^{5}$, Eduard Gratacós ${ }^{1,4}$ and Fatima Crispi ${ }^{1,4}$
}

BACKGROUND: Our aim was to determine the influence of breastfeeding and postnatal nutrition on cardiovascular remodeling induced by fetal growth restriction (FGR).

METHODS: A cohort study including 81 children with birthweight $<10$ th centile (FGR) and 121 with adequate fetal growth for gestational age (AGA) was conducted. Cardiovascular endpoints were left ventricular sphericity index (LVSI), carotid intima-media thickness (CIMT), and blood pressure (BP) at 4-5 $y$ of age. The combined effect of FGR and postnatal variablesincluding breastfeeding, fat dietary intake, and BMI-on cardiovascular endpoints was assessed by linear and robust regressions.

RESULTS: FGR was the strongest predictor of cardiovascular remodeling in childhood, leading to lower LVSI and increased CIMT and BP as compared with AGA. Breastfeeding $>6$ mo (coefficient: 0.0982) and healthy-fat dietary intake (coefficient: -0.0128) showed an independent beneficial effect on LVSI and CIMT, respectively. Overweight/obesity induced an additional increment of 1 SD on CIMT in FGR children (interaction coefficient: 0.0307) when compared with its effect in AGA. BMI increased systolic BP (coefficient: 0.7830) while weight catchup increased diastolic BP (coefficient: 4.8929).

CONCLUSIONS: Postnatal nutrition ameliorates cardiovascular remodeling induced by FGR. Breastfeeding and healthy-fat dietary intake improved while increased BMI worsened cardiovascular endpoints, which opens opportunities for targeted postnatal interventions from early life.

$\mathbf{T}^{\mathrm{T}}$ here is consistent epidemiological evidence that low birth weight is inversely associated with cardiovascular mortality in adulthood $(1,2)$. The most common cause of low birth weight is fetal growth restriction (FGR) due to placental insufficiency (3). FGR fetuses present cardiovascular remodeling already in utero that persist into childhood $(4,5)$. This effect is thought to contribute to their subsequent long-term cardiovascular risk (6). One of the most prominent signs of cardiac remodeling associated with FGR in fetal life and childhood is a more spherical heart measured by lower values on left ventricular sphericity index (LVSI) (5). Likewise, children who suffered FGR have signs of vascular dysfunction, including increased blood pressure (BP) (7) and carotid intima-media thickness (cIMT) (5) which are well-known cardiovascular risk factors.

Nutrition and body weight have a strong effect on cardiovascular development during childhood (8). Therefore, the effects of intrauterine environment on cardiovascular remodeling should be influenced by postnatal factors. Epidemiological studies suggest that obesity in childhood or accelerated postnatal growth could worsen the cardiovascular risk associated with impaired intrauterine growth. Moreover, high dietary intake of polyunsaturated fatty acids (PUFA) has been associated with lower cIMT values in children with FGR (9). However, the deleterious or protective effects of nutrition and body weight on cardiovascular remodeling have not been evaluated longitudinally in a cohort of FGR fetuses followed until childhood. In addition, the impact of breastfeeding on cardiac remodeling, which in general is considered a protective cardiovascular factor (10), has not been assessed specifically in children with FGR.

The aim of this study was to determine the independent and combined influence of intrauterine growth and postnatal nutrition on cardiovascular remodeling in childhood. For this, a multivariate regression analysis was performed to assess the effect of prenatal and postnatal environment on LVSI, CIMT, and BP within a cohort of 202 children including 81 FGR cases and 121 children with adequate fetal growth for gestational age (AGA).

\section{RESULTS}

\section{Characteristics of the Study Population}

A total of 202 children were evaluated on average at $4( \pm 1.6)$ years of age. Baseline and perinatal characteristics of the study populations are shown in Table 1. As expected, both groups showed significant differences in parental ethnicity, socioeconomic status, smoking, prevalence of steroids use, pregnancy complications, birth weight, and neonatal morbidity.

\footnotetext{
'BCNatal - Barcelona Center for Maternal-Fetal and Neonatal Medicine (Hospital Clínic and Hospital Sant Joan de Déu), IDIBAPS, University of Barcelona, Barcelona, Spain ${ }^{2}$ Epidemiology and Population Health Research Group (GESP), School of Public Health, Faculty of Health, Universidad del Valle, Cali, Colombia; ${ }^{3}$ Comfandi Health Services Research Group, Cali, Colombia; ${ }^{4}$ Centre for Biomedical Research on Rare Diseases (CIBER-ER), Barcelona, Spain; Institució Catalana de Recerca i Estudis Avançats (ICREA), Universitat Pompeu Fabra, Barcelona, Spain. Correspondence: Eduard Gratacós (gratacos@clinic.ub.es)

Received 26 March 2015; accepted 13 July 2015; advance online publication 7 October 2015. doi:10.1038/pr.2015.182
} 
Table 1. Baseline and perinatal characteristics of the study populations

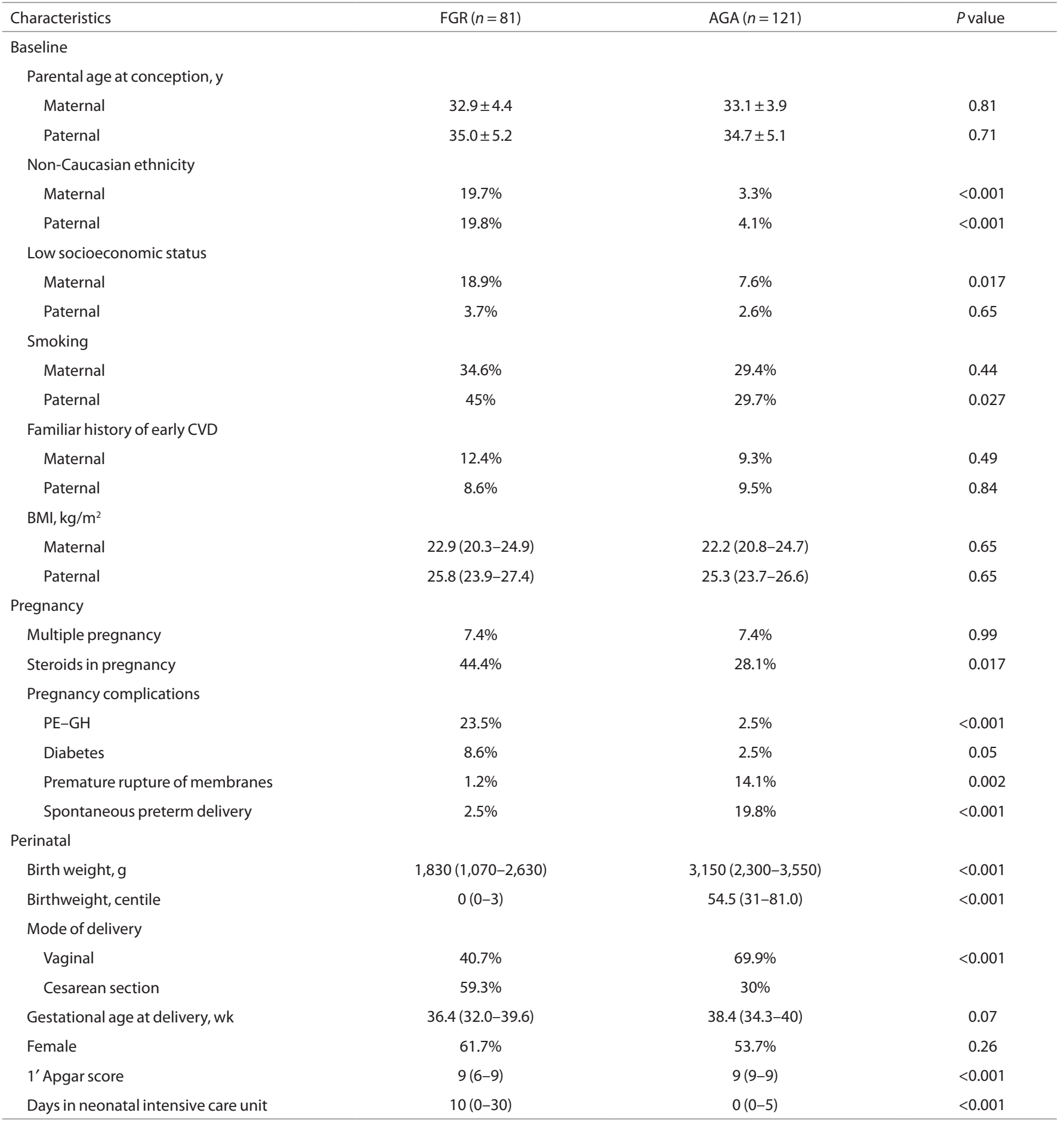

Data are percentage or mean \pm SD or median (interquartile range). AGA, adequate fetal growth for gestational age; $C V D$, cardiovascular disease; FGR, fetal growth restriction; $\mathrm{PE}-\mathrm{GH}$, preeclampsia-gestational hypertension.

Postnatal anthropometric and nutritional data are shown in Table 2. FGR children showed a lower height and weight at evaluation with a higher prevalence of catch-up growth as compared with AGA. The median duration of breastfeeding was 4 mo (interquartile range: $2-8$ ) similar in both groups. FGR children presented a similar dietary pattern as compared with
AGA, with the exception on higher percentage of protein intake and a nonsignificant trend to lower healthy-fat dietary intake.

The values of the cardiovascular endpoints used in this study are depicted in Table 3. There was a significant LVSI decrease and cIMT and BP increase in the FGR group as compared with AGA. 
Table 2. Postnatal anthropometric and nutritional characteristics of the study populations

\begin{tabular}{lccr}
\hline Characteristics & FGR $(n=81)$ & AGA $(n=121)$ & $P$ value \\
\hline Child age, $y$ & $4.2(2.3-4.9)$ & $5.0(2.6-5.6)$ & 0.002 \\
Height, $\mathrm{m}$ & $1.0(0.8-1.1)$ & $1.1(0.9-1.2)$ & $<0.001$ \\
Weight, kg & $15.2 \pm 4.5$ & $17.6 \pm 5.0$ & $<0.001$ \\
Weight catch-up & $88 \%$ & $65 \%$ & $<0.001$ \\
BMI, kg/m ${ }^{2}$ & $15.2(14.6-16.9)$ & $16.0(15.1-17.1)$ & 0.05 \\
Overweight-obese & $11.5 \%$ & $11.8 \%$ & 0.95 \\
Steroids in infancy & $8.8 \%$ & $4.1 \%$ & 0.17 \\
Breastfeeding & & & \\
Breastfeeding < 1 mo & $14.8 \%$ & $14.9 \%$ & 0.98 \\
Breastfeeding 1-6 mo & $56.8 \%$ & $57 \%$ & 0.97 \\
Breastfeeding $>$ 6 mo & $28.4 \%$ & $28.1 \%$ & 0.96 \\
Postnatal dietary characteristics & & & \\
\% Proteins & $18(16-19)$ & $16(15-19)$ & 0.03 \\
\% Carbohydrates & $49(43-52)$ & $48(45-53)$ & 0.77 \\
\% PUFA & $6.7(5.2-8.1)$ & $7.1(6.3-8.1)$ & 0.09 \\
\% Saturated fats & $38.9(33.9-42.3)$ & $39.2(35.5-40.8)$ & 0.95 \\
\% Unsaturated fats & $35.42(33.3-38.4)$ & $34.8(29.7-37.7)$ & 0.48 \\
Healthy-fat dietary intake & $15.7 \%$ & $25.8 \%$ & 0.11 \\
\hline Da & & & \\
\hline
\end{tabular}

Data are percentage or mean \pm SD or median (interquartile range). Healthy-fat dietary intake defined as polyunsaturated/saturated ratio $\geq 90$ th centile. AGA, adequate fetal growth for gestational age; FGR, fetal growth restriction; PUFA, polyunsaturated fatty acids.

Table 3. Cardiovascular endpoints in the study groups

\begin{tabular}{lccc}
\hline & FGR & AGA & \\
& $(n=81)$ & $(n=121)$ & $P$ value \\
\hline Left ventricular sphericity index & $1.36 \pm 0.15$ & $1.81 \pm 0.21$ & $<0.001$ \\
Carotid intima-media thickness, $\mathrm{mm}$ & $0.39 \pm 0.03$ & $0.37 \pm 0.03$ & $<0.001$ \\
Systolic blood pressure, $\mathrm{mmHg}$ & $106.05 \pm 7.53$ & $102.17 \pm 9.36$ & $<0.001$ \\
Diastolic blood pressure, $\mathrm{mmHg}$ & $70.30 \pm 6.53$ & $64.19 \pm 9.53$ & $<0.001$ \\
\hline
\end{tabular}

Data are mean \pm SD. $P$ values are adjusted by gestational age at delivery, gender, and child age. AGA, adequate fetal growth for gestational age; FGR, fetal growth restriction.

\section{Factors Associated to Postnatal Cardiovascular Endpoints}

FGR was the strongest predictor of LVSI (coefficient: -0.4038 (95\% confidence interval (CI): $-0.4610 ;-0.3467) ; P<0.001$ ). Regarding postnatal nutrition, prolonged breastfeeding (>6 mo) showed an independent positive association to LVSI (coefficient: 0.0982 (95\% CI 0.0133; 0.0183); $P=0.02$ ). There was a nonsignificant association with breastfeeding 1-6 mo (coefficient: 0.0484 (95\% CI: $-0.0279 ; 0.1247) ; P=0.21$ ). Neither healthy-fat dietary intake $(P=0.67)$ nor child BMI $(P$ $=0.64$ ) were statistically associated to LVSI. Regarding other factors, preeclampsia/gestational hypertension (coefficient: -0.0996 (95\% CI: $-0.1969 ;-0.0023) ; P=0.04)$ and child height (coefficient: 0.0040 (95\% CI: 0.0020; 0.0059); $P<0.001$ ) also showed a significant association to LVSI. Other baseline or perinatal characteristics (detailed in the Methods section) did not show a statistically significant association with LVSI.
FGR (coefficient: 0.0202 (95\% CI: 0.0112; 0.0296); $P<$ 0.001 ) and healthy-fat dietary intake (coefficient: -0.0128 (95\% CI: $-0.02473 ;-0.0011) ; P=0.03$ ) were significantly associated to cIMT. However, breastfeeding (1-6 mo: $P=$ 0.51 and $>6$ mo: $P=0.41$ ) and child BMI (coefficient: 0.0021 (95\% CI: $-0.0003 ; 0.0046) ; P=0.08$ ) were not statistically associated to cIMT. Regarding other factors, paternal smoking (coefficient: 0.0093 (95\% CI: 0.0002; 0.0184); $P=0.04$ ), child age (coefficient: 0.0037 (95\% CI: 0.0006; 0.0067); $P=$ 0.02 ), and gestational age at delivery (coefficient: -0.0020 (95\% CI: $-0.0032 ;-0.0008) ; P=0.001$ ) also showed a significant association to cIMT. Finally, other baseline or perinatal characteristics (detailed in the Methods section) did not show a statistically significant association with cIMT.

Both systolic BP (SBP; coefficient: 4.5801 (95\% CI: 1.6666; 7.4939); $P=0.002$ ) and diastolic BP (DBP; coefficient: 5.1975 (95\% CI: 2.3682; 8.0267); $P<0.001$ ) were positively associated to FGR. Regarding postnatal nutrition, child BMI was also positively associated to SBP (coefficient: 0.7830 (95\% CI: 0.0401; 1.5259); $P=0.04$ ), but not to DBP (coefficient: 0.647 (95\% CI: $-0.3566 ; 1.0861) ; P=0.30)$. Breastfeeding $(1-6$ mo: $P=0.56$ for SBP and $P=0.36$ for DBP; $>6$ mo: $P=0.54$ for SBP and $P=0.69$ for DBP) and healthy-fat dietary intake $(P=0.31$ for SBP and $P=0.68$ for DBP) were not statistically associated to BP. Regarding other characteristics, child's age was associated to SBP (coefficient: $1.7042(0.7937 ; 2.6147) ; P<0.001)$ and DBP (coefficient: $1.2341(0.3499 ; 2.1182) ; P=0.006)$, and weight catch-up to DBP (coefficient: 4.8929 (95\% CI: 1.3440; 8.4419); $P=0.007)$ but not with SBP $(P=0.14)$. Finally, other baseline or perinatal characteristics (detailed in the Methods section) did not show a statistically significant association with BP.

\section{Interaction Between FGR and Postnatal Nutrition on Cardiovascular Endpoints}

To assess any additional effect by the simultaneous presence of postnatal nutrition and FGR on cardiovascular remodeling, interaction coefficients were fitted. The effect of breastfeeding (interaction $P$ value $=0.44$ for breastfeeding $1-6$ mo, interaction $P=0.1$ for $>6 \mathrm{mo}$ ), healthy-fat dietary intake (interaction $P=0.30$ ), and BMI (interaction $P=0.24$ ) on LVSI was similar among AGA and FGR.

While the effect of breastfeeding (interaction $P=0.45$ for breastfeeding 1-6 mo, and interaction $P=0.42$ for $>6 \mathrm{mo}$ ) and healthy-fat dietary intake (interaction $P=0.64$ ) on cIMT was similar among AGA and FGR, a significant interaction between FGR and BMI could be observed. Being overweighted or obese produced a significant increment in $1 \mathrm{SD}$ on cIMT (interaction coefficient: 0.0307 (95\% CI: 0.0010; 0.0603); interaction $P=0.04$ ) within FGR population, but not in AGAs.

No significant interaction could be observed for breastfeeding (interaction $P=0.82$ and $P=0.72$ for breastfeeding 1-6 mo, and interaction $P=0.74$ and $P=0.62$ for breastfeeding $>6$ mo, for SBP and DBP, respectively), healthy-fat dietary intake ( $P=0.27$ and $P=0.12$, for SBP and DBP, respectively), and child BMI $(P=0.22$ and $P=0.22$, for SBP and DBP, respectively) and FGR on BP. 


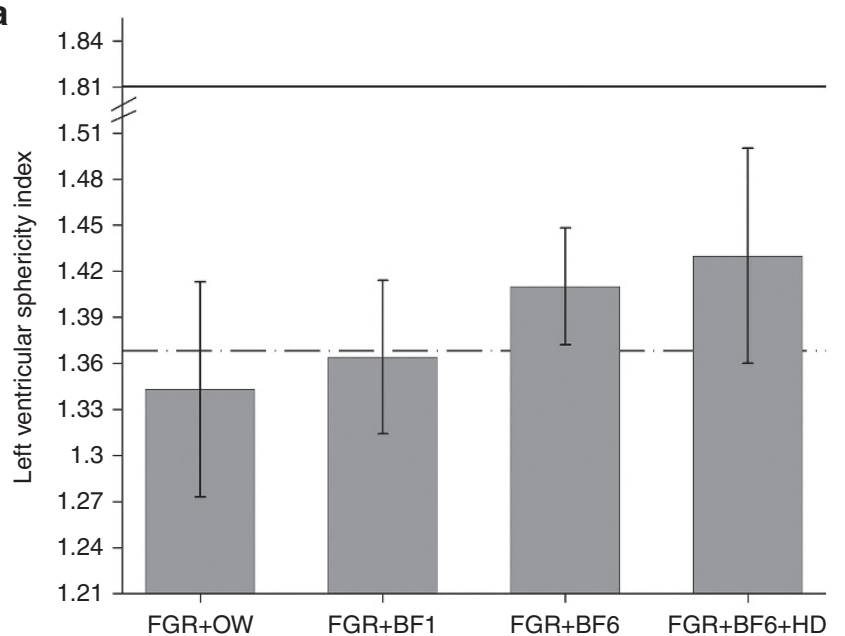

b

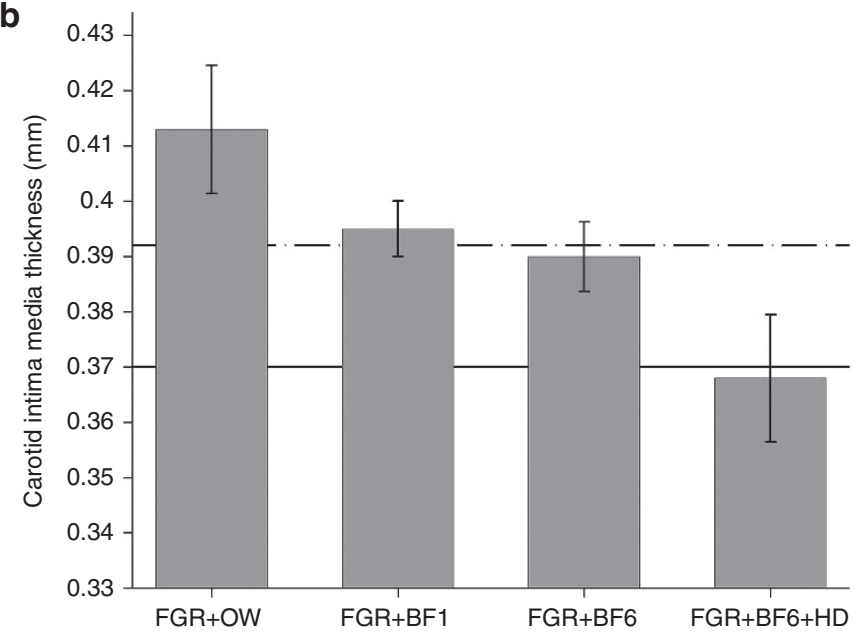

Figure 1. Effect of postnatal nutrition on cardiac and vascular remodeling in fetal growth restricted (FGR) children. Left ventricular sphericity index (a) and carotid intima-media thickness (b) marginal mean predictive values and SEs in the study populations. Solid line: mean value in normally grown children. Dashed line: mean value in FGR children. BF1, breastfeeding 1-6 mo; BF6, breastfeeding > $6 \mathrm{mo}$; HD, healthy-fat dietary intake; OW, overweight or obesity.

\section{Combined Effect of Postnatal Nutrition on LVSI and cIMT in FGR}

The combined effect induced by breastfeeding, healthy-fat dietary intake, and overweight/obesity on LVSI and cIMT in FGR children were assessed by calculating mean predictive values for LVSI and cIMT based on the final multivariate regression model described above and also including the interaction term between being overweight/obesity and FGR in the cIMT model. (Figure 1). Overweighted or obese FGR children had the worse LVSI and cIMT values, while those who breastfed $>6$ mo and had a healthy-fat dietary intake showed LVSI and cIMT values closer to AGA.

\section{DISCUSSION}

This study confirms the independent effect of intrauterine growth on cardiovascular programming. Besides FGR, we also confirm previous evidence on beneficial effect of high PUFA dietary intake on cIMT and further describe a protective effect of breastfeeding on cardiac remodeling of prenatal origin. Finally, a deleterious effect of overweight/obesity on cIMT and BP could be also demonstrated.

Our results reveal FGR as the strongest predictor of cardiac sphericity in childhood. These data are in line with the reported cardiac shape changes in fetuses and children with FGR $(4,5,11)$. In addition, breastfeeding was independently associated to more elongated (less remodeled) hearts, suggesting a potential beneficial effect of breastfeeding on cardiac structure in childhood. Interestingly, this protective effect could only be demonstrated by prolonged breastfeeding $(>6 \mathrm{mo})$, most probably due to the long time lapse needed for reverting cardiac remodeling. Our results are in agreement with the generation $\mathrm{R}$ study showing a significant impact of breastfeeding on postnatal cardiac mass at $6 \mathrm{y}$ of age childhood (12). The data are also consistent with animal studies suggesting that normal lactation may restore cardiomyocyte number after placental insufficiency (13). There is also literature indicating a protective effect of breastfeeding on cardiovascular risk factors such as hypertension, obesity, diabetes, and dyslipidemia (14). The potential underlying mechanisms linking breastfeeding with cardiac remodeling remains to be elucidated, but it may be related to growth factors, hormones, and favorable lipid profile of breast milk (15). It could also be mediated by the described slower pattern of growth (16), lower risk of overweight (17), and better endothelial function (18) in breast-fed as compared with formula-fed infants.

In contrast, we could not demonstrate any significant effect of healthy-fat dietary intake or child BMI on LVSI. No previous studies have evaluated the impact of PUFA intake or obesity on cardiac sphericity in FGR children. However, limited data from children with dilated cardiomyopathy (19) and animal models (20) suggest a potential protective effect of PUFA supplementation on ventricular remodeling. Regarding BMI, a variety of alterations in cardiac geometry that have been observed in obese children, being concentric and eccentric hypertrophy, the most commonly reported (21). Although our data suggest that the prenatal growth is a stronger predictor than obesity and diet for cardiac sphericity in early childhood, future studies in FGR are warranted to further assess the effect of BMI and PUFA intake on cardiac structure later in life. Regarding other perinatal factors, preeclampsia and gestational hypertension were also associated to cardiac sphericity in childhood. This is consistent with previous reports on cardiac remodeling in children born from preeclamptic mothers (22) and might be explained by the close interrelation among FGR, preeclampsia, and placental disease. Interestingly, other factors such as socioeconomic status, familiar history of cardiovascular disease, and catch-up growth could not demonstrate an independent effect on cardiac sphericity, emphasizing the expected direct impact of intrauterine environment on cardiac structure.

Regarding vascular remodeling, our results confirm previous data linking FGR $(23,24)$ with increased cIMT. In addition, our study also confirms the previously reported protective effect of high PUFA dietary intake on cIMT in childhood (25) and further demonstrates that this effect is independent 
from birthweight. In a recent clinical trial, supplemented $\omega-3$ PUFA from 6 mo to $5 \mathrm{y}$ of age prevented the inverse association between intrauterine growth and cIMT (26). Although it is unclear whether the vascular thickening is secondary to lipid deposition or adaptative to decrease wall stress, the beneficial effect of healthy fat on vasculature point to the antiinflammatory and anti-atherogenic properties of $\omega$-3 PUFA (27). Interestingly, the present study shows an additional effect of overweight/obesity on cIMT in FGR children. While this synergistic effect has been reported in adults (28), our results further indicate that it may occur already in childhood. As a reduction on lean tissue has been reported in FGR children (29), disproportionate high fat could produce an additional increment on vascular damage postnatally. In addition, we could not demonstrate any protective effect of breastfeeding on cIMT even after incorporating BMI in the model. Similar results were observed in the Young Finns cohort, where breastfeeding improved endothelial function but not cIMT (18). It is also consistent with the WHISTLER cohort study that could not demonstrate any benefit of breastfeeding on cIMT at $5 \mathrm{y}$ of age (30). Regarding other perinatal factors, we are also confirming previous data suggesting an association of prematurity (31) and paternal smoking (32) to cIMT.

Finally, BP was associated to intrauterine (FGR) and postnatal (child BMI and weight catch-up) growth and child's age with no significant association with breastfeeding or postnatal dietary intake. These results are consistent with previous reports demonstrating a relevant association between $\mathrm{BP}$ and FGR (33) and obesity in childhood (34). Interestingly, although a previous study showed the effect of growth catch-up on SBP (35), our results demonstrate its impact on DBP. Regarding breastfeeding, our study is also in accordance with the clusterrandomized PROBIT trial and the generation R cohort study, where no effect of breastfeeding on BP was found at $6 y$ of age (36). Finally, the potential interaction of postnatal diet and $\mathrm{BP}$ is controversial. Data from the generation $\mathrm{R}$ study suggest that macronutrient intake is not consistently associated to BP in childhood. On the contrary, the NHANES survey showed a protective effect of high dietary intakes of eicosapentaenoic acid and docosahexaenoic acid on SBP only among those children with low birth weight (37). This discordance may be explained by differences in the study populations and the type of nutritional questionnaires used in the studies. In this sense, we acknowledge that the design and limited sample size of our study may have prevented to demonstrate a potential association between $\mathrm{BP}$ and postnatal nutrition.

This study has several strengths and limitations. The study cohort was prospectively recruited including perinatal data as well as a comprehensive postnatal cardiovascular assessment (including echocardiography, vascular ultrasound, and BP measurement) and a dietary questionnaire. On the contrary, we acknowledge that other potentially predictive factors such as quantity and duration of smoking, exclusivity of breastfeeding, mother's diet, child's micronutrients quality, early adiposity rebound, or physical activity could not be assessed. The use of a 3 -d food record do not necessarily denote the variation in child intake across the year and it is not possible to infer a causal role of diet intake on cardiac remodeling as both were evaluated simultaneously. As foods habits can vary across populations, our results may not be directly extrapolated to other settings. Additionally, variability of BP measurements at one time point in children could limit the interpretation of BP results and warrants future follow-up studies. Besides, the limited sample size and the study design (with a high proportion of FGR cases included) may have prevented to demonstrate other interactions. Finally, our results cannot be directly extrapolated into adulthood. Therefore, future long-term studies are warranted to assess the impact of these findings in older ages.

In conclusion, we are confirming the strong effect of intrauterine environment on fetal programming and further demonstrate that postnatal nutrition could ameliorate subclinical cardiovascular remodeling. If confirmed by other studies, these data highlight the importance of public health strategies for promoting prolonged breastfeeding and healthy-fat dietary intake together with avoiding overweight and growth catchup for cardiovascular prevention. This is particularly important for those high-risk infants from pregnancies complicated by FGR, preeclampsia, or preterm delivery. Due to the high prevalence of these perinatal complications affecting $>10 \%$ of deliveries, such policies could have a high impact in public health. Further studies are warranted to define the optimal strategies to apply and select the populations that might better benefit from these interventions.

\section{METHODS}

\section{Study Design and Participants}

A prospective cohort study was conducted in 202 children selected from a delivery registry of a tertiary university hospital in Barcelona, Spain, between 2002 and 2007, and followed up into childhood. Exposed children were randomly selected among those with birth weight $<10$ th centile. Unexposed children were randomly selected from those children with adequate birth weight for gestational age (AGA) defined as birth weight $\geq 10$ th centile (38). A total of 81 FGR children were frequency matched by sex, gestational age at delivery $( \pm 2 \mathrm{wk})$ to 121 AGA children. Eighty percent of the children were already included in a previously published study (5). Congenital malformations, chromosomal defects, fetal infections, and monochorionic pregnancies were exclusion criteria. All newborns were managed by the same team and under the same protocol schemes in the ward and/or intensive care unit as appropriate. A written consent form was obtained from parents and the present study was approved by the ethical committees of Universidad del Valle (Colombia) and Hospital Clinic in Barcelona (Spain). Prenatal, perinatal, and postnatal information were obtained from clinical records, parental interview, and physical exams.

\section{Cardiovascular Endpoints}

The selected cardiovascular endpoints were LVSI, cIMT, and SBP and DBP. Cardiovascular measurements were done once during follow-up in childhood. Echocardiography and vascular ultrasound were performed according to a standardized protocol with a Siemens Sonoline Antares ultrasound system (Siemens Medical Systems, Malvern, PA) with 10-MHz phased-array and 13-MHz linear transducers. LVSI was calculated as base-to-apex length/basal diameter from an apical 4-chamber view at end-diastole. cIMT was measured at $1 \mathrm{~cm}$ proximal to the bifurcation in the right and left carotid offline based on a trace method with a computerized program (Siemens Syngo Arterial Health Package, Malvern, PA). BP was measured manually over the right brachial artery after 5-10 min 
resting by a trained nurse. SBP and DBP were obtained by the appearance of clear tapping and the completely disappearance of Korotkoff sound, respectively.

\section{Predictive Variables}

The main variables of this study were intrauterine growth, breastfeeding, postnatal PUFA dietary intake, and child BMI. Intrauterine growth was considered as a dichotomic variable into AGA (birth weight $\geq 10$ th centile) vs. FGR (birth weight $<10$ th centile). Breastfeeding information was obtained by parental interview and classified according to its duration into: $<1 \mathrm{mo}, 1-6 \mathrm{mo}$, and $>6 \mathrm{mo}$. Postnatal diet information was obtained from questionnaires filled at home by parents reporting the child's weighted food and beverages diaries consumed for $3 \mathrm{~d}$, previously used in Mediterranean Spanish population (39). The results were analyzed by the Diet source Junior 1.1.23 software (Nestle Health Care Nutrition S.A., Esplugues de Llobregat, Spain). Percentages of proteins, carbohydrates, and fats were calculated from total diet calorie consumptions; while saturated fatty acids, monounsaturated fatty acids, and PUFA percentages were calculated from total fat diet (the sum of three). Due to the significant inverse correlation between PUFA and saturated fatty acids ( $r$ $=-0.72$ ), their differential effect on cardiovascular risk, and the low consumption of PUFA in the study population, PUFA/saturated fatty acid ratio was generated and categorized as $<90$ (defined as unhealthyfat dietary intake) and $\geq 90$ percentile (defined as healthy-fat dietary intake). Anthropometric measurements in children were obtained by a trained nurse. Weight catch-up was defined when changes in the $Z$ score of the child weight compared with the $Z$ score at birth were higher than 0.67. $Z$ score was calculated using the default UK curves available in Stata.

Baseline, perinatal, and child anthropometric characteristics were also included in the analysis in order to assess their potential association with cardiovascular endpoints. Parental socioeconomic status, age, ethnicity, current BMI, smoking habits, and familiar history of cardiovascular disease were assessed by questionnaire. Low socioeconomic status was defined as routine occupation, long-term unemployment, or never worked, for both the pregnant woman and her partner. Early cardiovascular familiar history was defined by the presence of congenital heart disease, coronary artery disease, high BP, diabetes mellitus, or hypercholesterolemia in expanded first-degree pedigree (male $<55$ y old and female $<65$ y old). Perinatal data such as presence of gestational diabetes, preeclampsia, gestational hypertension, multiple pregnancy, premature rupture of membranes, spontaneous preterm delivery, prenatal or postnatal steroids, mode and gestational age at delivery, 5-min Apgar score, and days in neonatal intensive care unit were obtained from clinical record. Child age, height, weight, and weight catch-up were obtained at the time of the cardiovascular evaluation.

\section{Statistical Analysis}

Stata IC version 12.0 (StataCorp. LP, College Station, TX) was used for statistical analysis. Study groups were described and compared using mean (SD), median (interquartile range), or relative frequencies in tables with corresponding $t$-test or nonparametric and $\chi^{2}$ tests where appropriated. LVSI was arbitrarily chosen as a cardiac remodeling endpoint due to its strong association to FGR $(4,5)$ and its correlation to other echocardiographic parameters (Supplementary Data online).

Linear regression models were fitted including, in all cases, matching variables sex and gestational age at delivery (40). Main analysis was performed considering the classification of intrauterine growth (AGA vs. FGR) but the effect of birth weight percentile as a continuous variable was also explored (Supplementary Data online). Linear regression was applied in the case of LVSI and cIMT, while, due to the lack of assumption for linear regression, robust regression was applied for BP. Multivariate regression analyses were used to adjust for potential confounding and also to explore for interaction. A baseline model without interaction was fitted with those variables that in the univariate analysis yielded a $P \leq 0.20$ (results on univariate analysis included in the Supplementary Data online). A variable remain in the model when: partial $F$ had a $P$ $\leq 0.10$, confounding effect was observed, or by its clinical relevance on the outcome. Results from linear regression analyses are presented as regression coefficient with $95 \% \mathrm{CI}$ and their corresponding $P$ values.
In addition, one interaction term was added to the baseline model for each analysis to assess the modifying effects of breastfeeding duration, healthy-fat dietary intake, and being overweight-obese (defined as child BMI $\geq 85$ th centile) or normal, in the association of intrauterine growth and cardiovascular endpoints. The statistical significance of interaction was assessed with the Wald test and a $P$ value $<0.05$ was considered as statistically significant. Interaction coefficient quantifies the presence of a synergistic or antagonistic effect between postnatal nutrition and being FGR. Finally, based on multivariate analysis, marginal mean predictive values were generated for breastfeeding, overweight-obesity, and healthy-fat dietary intake in FGR children and presented in graphs.

\section{SUPPLEMENTARY MATERIAL}

Supplementary material is linked to the online version of the paper at http:// www.nature.com/pr

\section{ACKNOWLEDGMENTS}

M.R.-L. designed the study, conducted the data analysis, drafted the initial manuscript, and approved the final manuscript as submitted. L.O. designed the study, analyzed the data, co-wrote the initial manuscript, and approved the final manuscript as submitted. R.A. designed the study, contributed to acquisition of data, critically reviewed the manuscript, and approved the final manuscript as submitted. J.F. contributed to acquisition of data, critically reviewed the manuscript, and approved the final manuscript as submitted. M.C.-L. contributed to acquisition of data, critically reviewed the manuscript, and approved the final manuscript as submitted. F.F. designed the study, contributed to interpretation of the results, critically reviewed the manuscript, and approved the final manuscript as submitted. B.B. contributed to interpretation of the results, critically reviewed the manuscript, and approved the final manuscript as submitted. E.G. designed the study, contributed to interpretation of the results, critically reviewed the manuscript, and approved the final manuscript as submitted. F.C. designed the study, contributed to acquisition of data, interpretation of the results, critically reviewed the manuscript, and approved the final manuscript as submitted.

\section{STATEMENT OF FINANCIAL SUPPORT}

This project has been funded with support of the Universidad del Valle (Colombia), Erasmus + Programme of the European Union (Framework Agreement number: 2013-0040), and by grants from Instituto de Salud Carlos III and Ministerio de Economia y Competitividad (ref. PI11/00051, $\mathrm{PI} 11 / 01709, \mathrm{Pl} 12 / 00801, \mathrm{PI} 14 / 00226$, and SAF2012-37196) cofinanciado por el Fondo Europeo de Desarrollo Regional de la Unión Europea "Una manera de hacer Europa," Centro para el Desarrollo Técnico Industrial (CENIT 20092012, apoyado por el Ministerio de Ciencia e Innovación, y Fondo de inversión local para el empleo; Spain), the Fundación Mutua Madrileña, Fundació Agrupació Mutua, Obra Social "la Caixa," and Cerebra Foundation for the Brain Injured Child (Carmarthen, Wales, UK). This publication reflects the views only of the author, and the Commission cannot be held responsible for any use which may be made of the information contained therein.

Disclosure: The authors declare that there are not any financial and nonfinancial competing interests. The authors indicate that we do not have any financial relationships relevant to this article to disclose.

\section{REFERENCES}

1. Barker DJ. Fetal origins of cardiovascular disease. Ann Med 1999;31:Suppl 1:3-6.

2. Risnes KR, Vatten LJ, Baker JL, et al. Birthweight and mortality in adulthood: a systematic review and meta-analysis. Int J Epidemiol 2011;40:64761.

3. Figueras F, Gratacós E. Update on the diagnosis and classification of fetal growth restriction and proposal of a stage-based management protocol. Fetal Diagn Ther 2014;36:86-98.

4. Cruz-Lemini M, Crispi F, Valenzuela-Alcaraz B, et al. A fetal cardiovascular score to predict infant hypertension and arterial remodeling in intrauterine growth restriction. Am J Obstet Gynecol 2014;210:e1-552.e22. 
5. Crispi F, Bijnens B, Figueras F, et al. Fetal growth restriction results in remodeled and less efficient hearts in children. Circulation 2010;121: 2427-36.

6. Demicheva E, Crispi F. Long-term follow-up of intrauterine growth restriction: cardiovascular disorders. Fetal Diagn Ther 2014;36:143-53.

7. Shankaran S, Das A, Bauer CR, et al. Fetal origin of childhood disease: intrauterine growth restriction in term infants and risk for hypertension at 6 years of age. Arch Pediatr Adolesc Med 2006;160:977-81.

8. Kuklina EV. Breastfeeding and cardiometabolic profile in childhood: how infant feeding, preterm birth, socioeconomic status, and obesity may fit into the puzzle. Circulation 2014;129:281-4.

9. Skilton MR, Mikkilä V, Würtz P, et al. Fetal growth, omega-3 (n-3) fatty acids, and progression of subclinical atherosclerosis: preventing fetal origins of disease? The Cardiovascular Risk in Young Finns Study. Am J Clin Nutr 2013;97:58-65.

10. Agostoni C, Braegger C, Decsi T, et al. Breast-feeding: a commentary by the ESPGHAN Committee on Nutrition. J Pediatr Gastroenterol Nutr 2009;49:112-25.

11. Crispi F, Figueras F, Cruz-Lemini M, Bartrons J, Bijnens B, Gratacos E. Cardiovascular programming in children born small for gestational age and relationship with prenatal signs of severity. Am J Obstet Gynecol 2012;207:121.e1-9.

12. de Jonge LL, Langhout MA, Taal HR, et al. Infant feeding patterns are associated with cardiovascular structures and function in childhood. J Nutr 2013;143:1959-65.

13. Black MJ, Siebel AL, Gezmish O, Moritz KM, Wlodek ME. Normal lactational environment restores cardiomyocyte number after uteroplacental insufficiency: implications for the preterm neonate. Am J Physiol Regul Integr Comp Physiol 2012;302:R1101-10.

14. Owen CG, Whincup PH, Cook DG. Breast-feeding and cardiovascular risk factors and outcomes in later life: evidence from epidemiological studies. Proc Nutr Soc 2011;70:478-84.

15. Martin RM, Davey Smith G. Does having been breastfed in infancy influence lipid profile in later life?: a review of the literature. Adv Exp Med Biol 2009;646:41-50.

16. Ong KK, Preece MA, Emmett PM, Ahmed ML, Dunger DB; ALSPAC Study Team. Size at birth and early childhood growth in relation to maternal smoking, parity and infant breast-feeding: longitudinal birth cohort study and analysis. Pediatr Res 2002;52:863-7.

17. Oddy WH, Mori TA, Huang RC, et al. Early infant feeding and adiposity risk: from infancy to adulthood. Ann Nutr Metab 2014;64:262-70.

18. Järvisalo MJ, Hutri-Kähönen N, Juonala M, et al. Breast feeding in infancy and arterial endothelial function later in life. The Cardiovascular Risk in Young Finns Study. Eur J Clin Nutr 2009;63:640-5.

19. Firuzi O, Shakibazad N, Amoozgar H, et al. Effects of omega-3 polyunsaturated fatty acids on heart function and oxidative stress biomarkers in pediatric patients with dilated cardiomyopathy. Int Cardiovasc Res J 2013;7:8-14.

20. Duda MK, O'Shea KM, Lei B, et al. Dietary supplementation with omega-3 PUFA increases adiponectin and attenuates ventricular remodeling and dysfunction with pressure overload. Cardiovasc Res 2007;76:303-10.

21. Mangner N, Scheuermann K, Winzer E, et al. Childhood obesity: impact on cardiac geometry and function. JACC Cardiovasc Imaging 2014;7: 1198-205.

22. Davis EF, Lazdam M, Lewandowski AJ, et al. Cardiovascular risk factors in children and young adults born to preeclamptic pregnancies: a systematic review. Pediatrics 2012;129:e1552-61.

23. Stergiotou I, Crispi F, Valenzuela-Alcaraz B, Cruz-Lemini M, Bijnens B, Gratacos E. Aortic and carotid intima-media thickness in term small-for- gestational-age newborns and relationship with prenatal signs of severity. Ultrasound Obstet Gynecol 2014;43:625-31.

24. Skilton MR, Viikari JS, Juonala M, et al. Fetal growth and preterm birth influence cardiovascular risk factors and arterial health in young adults: the Cardiovascular Risk in Young Finns Study. Arterioscler Thromb Vasc Biol 2011;31:2975-81.

25. Ayer JG, Harmer JA, Xuan W, et al. Dietary supplementation with n-3 polyunsaturated fatty acids in early childhood: effects on blood pressure and arterial structure and function at age 8 y. Am J Clin Nutr 2009;90: $438-46$.

26. Skilton MR, Ayer JG, Harmer JA, et al. Impaired fetal growth and arterial wall thickening: a randomized trial of $\omega-3$ supplementation. Pediatrics 2012;129:e698-703.

27. Sudheendran S, Chang CC, Deckelbaum RJ. N-3 vs. saturated fatty acids: effects on the arterial wall. Prostaglandins Leukot Essent Fatty Acids 2010;82:205-9.

28. Barker DJ, Osmond C, Kajantie E, Eriksson JG. Growth and chronic disease: findings in the Helsinki Birth Cohort. Ann Hum Biol 2009;36: $445-58$.

29. Singhal A, Wells J, Cole TJ, Fewtrell M, Lucas A. Programming of lean body mass: a link between birth weight, obesity, and cardiovascular disease? Am J Clin Nutr 2003;77:726-30.

30. Evelein AM, Geerts CC, Visseren FL, et al. The association between breastfeeding and the cardiovascular system in early childhood. Am J Clin Nutr 2011;93:712-8.

31. Lewandowski AJ, Augustine D, Lamata P, et al. Preterm heart in adult life: cardiovascular magnetic resonance reveals distinct differences in left ventricular mass, geometry, and function. Circulation 2013;127: 197-206.

32. Gall S, Huynh QL, Magnussen CG, et al. Exposure to parental smoking in childhood or adolescence is associated with increased carotid intimamedia thickness in young adults: evidence from the Cardiovascular Risk in Young Finns study and the Childhood Determinants of Adult Health Study. Eur Heart J 2014;35:2484-91.

33. Mu M, Wang SF, Sheng J, et al. Birth weight and subsequent blood pressure: a meta-analysis. Arch Cardiovasc Dis 2012;105:99-113.

34. Friedemann C, Heneghan C, Mahtani K, Thompson M, Perera R, Ward AM. Cardiovascular disease risk in healthy children and its association with body mass index: systematic review and meta-analysis. BMJ 2012;345:e4759.

35. Hemachandra AH, Howards PP, Furth SL, Klebanoff MA. Birth weight, postnatal growth, and risk for high blood pressure at 7 years of age: results from the Collaborative Perinatal Project. Pediatrics 2007;119: e1264-70.

36. Kramer MS, Fombonne E, Igumnov S, et al. Effects of prolonged and exclusive breastfeeding on child behavior and maternal adjustment: evidence from a large, randomized trial. Pediatrics 2008;121:e435-40.

37. Skilton MR, Raitakari OT, Celermajer DS. High intake of dietary longchain $\omega-3$ fatty acids is associated with lower blood pressure in children born with low birth weight: NHANES 2003-2008. Hypertension 2013;61:972-6.

38. Figueras F, Meler E, Iraola A, et al. Customized birthweight standards for a Spanish population. Eur J Obstet Gynecol Reprod Biol 2008;136:20-4.

39. Schröder H, Covas MI, Marrugat J, et al. Use of a three-day estimated food record, a 72-hour recall and a food-frequency questionnaire for dietary assessment in a Mediterranean Spanish population. Clin Nutr 2001;20:429-37.

40. Sjölander A, Greenland S. Ignoring the matching variables in cohort studies - when is it valid and why? Stat Med 2013;32:4696-708. 